\title{
A novel fast and accurate pseudo-analytical simulation approach for MOAO
}

\author{
E. Gendron ${ }^{a}$, A. Charara ${ }^{b}$, A. Abdelfattah ${ }^{b}$, D. Gratadour ${ }^{a}$, D. Keyes ${ }^{b}$, H. Ltaief $^{b}$, C. Morel $^{a}$, \\ F. Vidal ${ }^{a}$, A. Sevin ${ }^{a}$ and G. Rousset ${ }^{a}$ \\ ${ }^{a}$ LESIA, Observatoire de Paris, CNRS, UPMC, Université Paris Diderot, 5 place J. Janssen, \\ 92190 Meudon, France \\ ${ }^{b}$ Extreme Computing Research Center, King Abdullah University of Science and Technology, \\ 23955 Thuwal, Kingdom of Saudi Arabia
}

\begin{abstract}
Multi-object adaptive optics (MOAO) is a novel adaptive optics (AO) technique for wide-field multi-object spectrographs (MOS). MOAO aims at applying dedicated wavefront corrections to numerous separated tiny patches spread over a large field of view (FOV), limited only by that of the telescope. The control of each deformable mirror (DM) is done individually using a tomographic reconstruction of the phase based on measurements from a number of wavefront sensors (WFS) pointing at natural and artificial guide stars in the field.

We have developed a novel hybrid, pseudo-analytical simulation scheme, somewhere in between the end-toend and purely analytical approaches, that allows us to simulate in detail the tomographic problem as well as noise and aliasing with a high fidelity, and including fitting and bandwidth errors thanks to a Fourier-based code.

Our tomographic approach is based on the computation of the minimum mean square error (MMSE) reconstructor, from which we derive numerically the covariance matrix of the tomographic error, including aliasing and propagated noise. We are then able to simulate the point-spread function (PSF) associated to this covariance matrix of the residuals, like in PSF reconstruction algorithms.

The advantage of our approach is that we compute the same tomographic reconstructor that would be computed when operating the real instrument, so that our developments open the way for a future on-sky implementation of the tomographic control, plus the joint PSF and performance estimation.

The main challenge resides in the computation of the tomographic reconstructor which involves the inversion of a large matrix (typically $40000 \times 40000$ elements). To perform this computation efficiently, we chose an optimized approach based on the use of GPUs as accelerators and using an optimized linear algebra library: MORSE providing a significant speedup against standard CPU oriented libraries such as Intel MKL. Because the covariance matrix is symmetric, several optimization schemes can be envisioned to speedup even further the computation. Optimizing the speed of the reconstructor computation is of major interest not only for the design study of MOAO instruments, but also for future routine operations of the system as the reconstructor has to be updated regularly to cope for atmospheric variability.
\end{abstract}

Keywords: adaptive optics, wavefront sensing, high performance computing, hardware accelerators

\section{INTRODUCTION}

One of the main scientific drivers of the European-Extremely Large Telescope (E-ELT) is the study of distant galaxies, and a multi-object spectrograph (MOS) is a key instrument for this purpose. As those galaxies are small (typ. $0.2-0.5$ arcsec) they require the use of adaptive optics $(\mathrm{AO})^{1,2}$ : this is how multi-object AO (MOAO) was born in $2003^{3}$, when people first tried to couple AO to MOS.

The current E-ELT project called MOSAIC ${ }^{4,5}$, supported by a European consortium, is a proposal for such an instrument. MOSAIC would be installed on the E-ELT, of $39 \mathrm{~m}$ diameter, at the horizon 2020. It is a nearinfrared multi-object spectrograph with about 20 channels equipped with adaptive optics ${ }^{2}$. MOAO is using

Further author information: eric.gendron at obspm.fr 
tomography for driving the adaptive deformable mirrors (DM), and thus requires several wavefront-sensors. The wavefront sensing would make use of 6 laser guide stars and up to 5 natural ones (up to 11 WFS in total). Due to the large size of the telescope, the number of sub-apertures of each WFS across the pupil diameter would be in the range $60-80$, possibly larger. The total number of measurements is then in the range $66 \mathrm{~K}-110 \mathrm{~K}$. The computation of the tomographic reconstructor then involves the inversion of matrices of size up to $110 \mathrm{~K} \times 110 \mathrm{~K}$.

We propose in this paper a method for simulating the tomographic performance of such a system in order to dimension it properly. Our method is gathering ideas borrowed to a number of various simulation techniques, such as PSF reconstruction, Fourier analysis, end-to-end simulation codes and real systems. First, we're going to use a matrix formalism to compute the covariance matrices of the residual tomographic error. From that, we will apply a PSF reconstruction process in order to get the phase structure function of the tomographic error, including aliasing and noise. Then, we will get the other phase structure functions (fitting error, bandwidth) using a Fourier approach, i.e. quite a classical method now, as already described in $^{6-8}$. In order to push the PSF reconstruction process to the scale of an ELT, we have developed a kind of simplified method, that potentially could be used for PSF reconstruction on the E-ELT using the real covariance matrices measured on-sky. All this is explained in section 3.4 .

Now, the covariance matrices of tomographic error are treated using a full linear algebra formalism, based on the matrix of the tomographic reconstructor. In order to get best performance, we will use MMSE reconstructors, that require the computation of the inverse of the covariance matrix of the measurements. This compute intensive procedure relies on the Matrix Over Runtime System at Exascale (MORSE) library. This high productivity library allows to efficiently schedule various computational tasks and to fully exploit the underlying complex heterogeneous architectures (x86 many core system with hardware accelerators). This is the key of this simulation, without which it would not be possible. All this is explained in section 5 .

Another delicate point in the generation of the covariance matrices. Those are usually computed using matrix operators linking the modal expansions of the phase between the layer-projected meta-pupils, which makes the computations heavier with the size of the system. Instead, we propose here compute each covariance coefficient in a simplified analytical way, just as done in the CANARY demonstrator experiment for fitting theoretical covariance matrices to on-sky ones. Computing each matrix element in a individual way opens the way to parallelization. This is described in Sect. 4.

We will then give some examples at the end of the paper.

\section{SYSTEM DESCRIPTION}

We simulate here a system with an arbitrary combination number of LGS and NGS. We simulate a tomographic system optimizing the reconstruction along a single direction of interest, i.e. MOAO or LTAO. We will use the term "on-axis" to name this optimization direction. The tomographic error is simulated as if the system was working in open-loop. The temporal aspects will enter in the bandwidth error term (Sect. 3.3), and only this term makes a difference between an MOAO or LTAO system in our analysis.

We will assume that the system is performing the tomographic reconstruction using a minimum mean square error (MMSE) reconstructor, that will reconstruct the measurements that would be seen by a factious, noiseless WFS staring at the target to be compensated for.

\subsection{The tomographic reconstructor}

A MMSE approach has been used as soon as the 80 's to reconstruct the wavefront ${ }^{9}$, and then for tomography ${ }^{10,11}$. Briefly summarized, if $\vec{m}$ is a measurement vector (including noise, etc) and $\vec{t}$ the quantity to be retrieved, the linear application that minimizes the norm of $|\vec{t}-R \vec{m}|^{2}$ (or more generally the scalar product $\left.(\vec{t}-R \vec{m})^{t} \cdot \Delta \cdot(\vec{t}-R \vec{m})\right)$ is given by :

$$
R=C_{t m} \cdot C_{m m}^{-1}
$$

where $C_{m m}$ stands for the covariance matrix between all the measurements of all the WFS of the instrument, and $C_{t m}$ is the covariance matrix between $\vec{t}$ (the measurements of the factious truth sensor in our case), and all the other system measurements. This is the approach we will follow here. The efficient computation of the 
covariance matrices will be described in Sect. 4, and their delicate inversion in Sect.5. It is emphasized that the instrument measurements englobes not only the turbulence effects, but also the noise: they are the sum of purely turbulent matrices, and noise covariance matrices.

\subsection{The CANARY approach}

The "factious truth sensor" approach we describe here is not just fiction. It is the approach followed by the demonstrator instrument called CANARY. CANARY is a demonstrator for MOAO ${ }^{11-14}$, that has been set up on the $4.2 \mathrm{~m}$ William Herschel Telescope. It has demonstrated as soon as 2010 successful tomographic compensation of a single star in open-loop, measuring off-axis wavefronts on NGS located as far as 50" off-axis. Its tomographic reconstructor is computed as described in the previous paragraph, for a particular reason : CANARY is equipped with a so-called truth sensor, the equivalent of our factious WFS, which is sensing the on-axis wavefront (for the only purpose of calibration and diagnostics, and of course without taking any part to the compensation). This on-axis WFS allows us to calibrate for the interaction matrix on the bench, which inverse will allows us to drive the DM by minimizing the calibration errors from the tomographic information. If we call $\vec{t}$ the measurements of the truth sensor and $\vec{v}$ the voltages applied on the DM, we can calibrate the interaction matrix $D$ by poking one by one each actuator :

$$
\vec{t}=D \vec{v}
$$

and we can get a way to control the DM from the TS measurements using

$$
\vec{v}=D^{\dagger} \vec{t}
$$

where $D^{\dagger}$ is the generalized inverse of $D$, possibly with some filtered modes. $D^{\dagger}$ is usually computed by doing a singular value decomposition (SVD) of $D=U \cdot\left[\operatorname{diag}\left(\lambda_{i}\right)\right] \cdot V^{t}$, and inverting all eigenvalues but the few weakest ones

$$
D^{\dagger}=V \cdot\left[\operatorname{diag}\left(\lambda_{i}^{-1}-0\right)\right] \cdot U^{t}
$$

This approach is sub-optimal, since it is made of 2 steps: an optimal one using the MMSE estimator $R$ (Eq. 1), followed by a second, least-square step, using $D^{\dagger}$. The advantage of doing so is to be able to actually calibrate this second step and thus avoid model error, while the first one can be left purely theoretical.

\subsection{Computation of the tomographic error}

The tomographic error vector $\vec{e}$, as it would be measured by a noiseless truth sensor, would be

$$
\vec{e}=\vec{t}-R \vec{m}
$$

We can compute the covariance matrix $C_{e e}$ of the tomographic error vector $\vec{e}$. It comes

$$
C_{e e}=C_{t t}-C_{t m} R^{t}-R C_{t m}^{t}+R C_{m m} R^{t}
$$

and we can see that replacing $R$ by its expression given in Eq. 1 shows that the computation of matrix $C_{e e}$ can be based on the covariance matrices $C_{t t}, C_{t m}$ and $C_{t m}$, which are all covariance matrices of WFS measurements. Notice that the above equation could be simplified, developing the expression of $R$. We prefer to keep this general expression, which remains valid for any matrix $R$, MMSE or else.

In particular, we can imagine that $R$ could be computed using a slightly different $C_{n}^{2}(h)$ turbulence profile from that used in the computation of $C_{e e}$. Indeed, it is a particularly interesting topic to study the effects of $C_{n}^{2}(h)$ profile variations on the correction, as this is likely to occur on-sky in the real case. This equation allows to cope with this.

In addition, it must be noticed that the inversion of matrix $C_{m m}$ in Eq. 1 is not a real inversion, as $C_{m m}$ may contain null eigenvalues. Inverting $C_{m m}$ may be done using diagonalization, and filtering of the weakest eigenvalues. Hence, the product $C_{m m}^{-1} C_{m m} \neq \mathrm{Id}$. 


\section{SIMULATION}

\subsection{Goal}

We aim to simulate the image quality attained on the EELT using MOAO. For this we aim to simulate a long-exposure point spread function (PSF). The long-exposure PSF is the Fourier transform (noted $\mathcal{F}[\ldots]$ in the equation below) of the optical transfer function (OTF). Under hypothesis of stationarity of the phase, it has been shown ${ }^{15}$ that the PSF can be written as

$$
P S F=\mathcal{F}[O T F(\rho / \lambda)]=\mathcal{F}\left[O T F_{\text {tel }}(\rho / \lambda) \exp \left(-\frac{1}{2} D_{\phi}(\rho)\right)\right]
$$

with $O T F_{\text {tel }}$ the optical transfer function of the telescope, and $D_{\phi}(\rho)$ the structure function of the residual phase. We will assume that the residual errors induced by the AO correction will be made of 3 independent terms :

- a term made of the tomographic error, the associated aliasing, and the noise propagated from the measurements through the tomographic reconstructor

- a term due to temporal error, due to the finite system bandwidth,

- a term due to the DM fitting error

A structure function will be associated to each of these terms, that will be computed from the power spectral density of the residual phase for the 2 last items, exactly as proposed in ${ }^{6-8}$. We will assume that these 3 terms behave as independent processes so that the structure function of the residual phase can be written as the following sum :

$$
D_{\phi}(\rho)=D_{\text {fit }}(\rho)+D_{\text {bw }}(\rho)+D_{\text {tomo }}(\rho)
$$

Of course, other error contributors can be added to this list if their structure function is known. Anyway, the detail of the computation of these 3 terms is detailed in the 3 following paragraphs.

\subsection{Computation of $D_{\text {fit }}(\rho)$}

The relation between structure function of the phase and the spectrum $W(k)$ of the same phase is given by

$$
D_{\text {fit }}(\rho)=2 \iint_{|k|>f_{D M}} W(\vec{k})(1-\cos (2 \pi \vec{k} \vec{\rho})) d^{2} \vec{k}
$$

The spectrum $W(\vec{k})$ is the Von-Karman spectrum :

$$
W(\vec{k})=0.023 r_{0}^{-5 / 3}\left(k^{2}+1 / L_{0}^{2}\right)^{-11 / 6}
$$

and the integration is done over frequencies located out of the domain of the DM. For a DM with square geometry of actuators, this domain corresponds to frequencies with $\left|k_{x}\right|>f_{c}$ (and same for $y$ ), with $f_{c}=1 / 2 . d_{\text {act }}$.

\subsection{Computation of $D_{\mathrm{bw}}(\rho)$}

The structure function of the bandwidth error of the system can be computed assuming that the wavefront translates in front of the pupil, and that this translation is the only cause for the temporal evolution. The spatial frequencies $\vec{k}$ can be turned into temporal frequencies $f$ using $\vec{k} \cdot \vec{V}=f$, and the power spectrum of the residual phase can be computed by applying to the phase spectrum at a given spatial frequency $W(\vec{k})$ a factor $H_{\text {rej }}(\vec{k} \cdot \vec{V})$, with $H_{r e j}(f)$ the rejection transfer function of the adaptive optics (that may have a different expression in MOAO and LTAO depending on the open- or closed-loop scheme). This approach can be followed for each layer, with the particular wind speed $\vec{V}_{l}$ associated to each layer.

This approach assumes that the wind speed $\vec{V}_{l}$ has a particular fixed direction, and it usually results in a significantly elongated PSF when the $\vec{V}_{l}$ are co-aligned or when only a single layer is simulated. To the contrary, it is known and observed that, in reality, the wind direction significantly fluctuates and the boiling part is 
important enough so that PSFs are most of the time symmetric. That's why we have decided to model the wind speed as a vector $\vec{V}$ which direction is changing arbitrarily, so that in average the spatial frequencies are linked to temporal ones using $k V / \sqrt{2}=f$ ( $k$ and $V$ are the modulus of their associated vectors).

The spectrum of the residual phase of the temporal errors is then :

$$
W_{\mathrm{bw}}(\vec{k})=W(\vec{k}) H_{\mathrm{rej}}(k V / \sqrt{2})
$$

\subsection{Computation of $D_{\text {tomo }}(\rho)$}

Computing this term will be done using a matrix formulation, in order to derive the phase properties exactly as the system does. The structure function of the phase tomographic error $D_{\text {tomo }}(\rho)$ will be deduced from the statistical covariance matrix of the DM actuators, $C_{v v}$ (the underscript $v$ stands for volts). The matrix $C_{v v}$ is computed using

$$
C_{v v}=D^{\dagger} C_{e e} D^{\dagger t}
$$

with $C_{e e}$ given in Eq. 6 and $D^{\dagger}$ explained at Eq. 4. We write the phase produced by the DM as

$$
\phi(\vec{x})=\sum_{i} v_{i} f_{i}(\vec{x})
$$

with $f_{i}(\vec{x})$ the influence functions of the actuators, and $\vec{x}$ the vector of spatial coordinates in the pupil. The general expression of the phase structure function is then

$$
\begin{aligned}
D_{\text {tomo }}(\vec{x}, \vec{\rho}) & =<(\phi(\vec{x})-\phi(\vec{x}+\vec{\rho}))^{2}> \\
& =<\left[\sum_{i=1}^{N} v_{i} f_{i}(\vec{x})-\sum_{j=1}^{N} v_{j} f_{j}(\vec{x}+\vec{\rho})\right]^{2}>
\end{aligned}
$$

We will develop this equation, and introduce a first hypothesis : we will consider that the phase structure function is invariant, i.e. does not depend on variable $\vec{x}$ : then, we will average the function over $\vec{x}$, as initially done in (Véran et al., 1997), ${ }^{16}$ or in next article such as (Gendron et al., 2006) ${ }^{17}$. Averaging means integrate over $\vec{x}$ and normalize to the domain area it's been integrated over. This domain area is the pupil autocorrelation

$$
G(\vec{\rho})=\int P(\vec{x}) P(\vec{x}+\vec{\rho}) d^{2} \vec{x}
$$

Developing the square of a difference in Eq. 15 leads to 3 terms : the two squares, and the double product. Integrating over $\vec{x}$ reduces the two squares to a same expression. It finally comes

$$
D_{\text {tomo }}(\vec{\rho})=\frac{2}{G(\vec{\rho})} \sum_{i, j}<v_{i} v_{j}>\int f_{i}(\vec{x}) f_{j}(\vec{x}) d \vec{x}-\frac{2}{G(\vec{\rho})} \sum_{i, j}<v_{i} v_{j}>\int f_{i}(\vec{x}) f_{j}(\vec{x}+\vec{\rho}) d x
$$

The above expression is approximated : there is a missing term $P(x) P(x+\rho)$ under both integrals. We have chosen to drop this term, because the phase we're dealing with is given by Eq.13 and quickly tends towards 0 anyway outside the pupil $P(\vec{x})$, simply because there is no actuator outside it. Hence, we decide to ignore the multiplication by the pupil mask $P(\vec{x})$ : this may lead to approximations of the phase close to the pupil edges.

Then, a third approximation is now to write that all the influence functions are all identical to a master one $f(\vec{x})$, deduced from it by a translation, i.e. $f_{i}(\vec{x})=f\left(\vec{x}-\overrightarrow{x_{i}}\right)$. We can imagine the function $f(\vec{x})$ is centered on the pupil, and $\overrightarrow{x_{i}}$ is just the position vector of actuator number $i$. We also introduce the vector $\overrightarrow{x_{i j}}=\overrightarrow{x_{i}}-\overrightarrow{x_{j}}=-\overrightarrow{x_{j i}}$, which is the separation vector between 2 actuators $i$ and $j$. In order to lighten the calculus, we will split Eq. 17 into the two terms and write

$$
D_{\text {tomo }}(\vec{\rho})=2 C(\overrightarrow{0})-2 C(\vec{\rho})
$$

We will introduce now the autocorrelation function of $f(\vec{x})$. It is given by

$$
A(\vec{\rho})=\int f(\vec{x}) f(\vec{x}+\vec{\rho}) d^{2} \vec{x}
$$


and it will allow us to rewrite the expression of $C(\vec{\rho})$ as

$$
\begin{aligned}
C(\vec{\rho}) & =\frac{1}{G(\rho)} \sum_{i, j}<v_{i} v_{j}>\int f\left(x-x_{i}\right) f\left(x-x_{j}+\rho\right) d x \\
& =\frac{1}{G(\rho)} \sum_{i, j}<v_{i} v_{j}>A\left(\overrightarrow{x_{i j}}+\vec{\rho}\right)
\end{aligned}
$$

We will now introduce the quantity $c_{v}\left(\overrightarrow{x_{i j}}\right)$ : this is the average covariance term $\left\langle v_{i} v_{j}\right\rangle$ for all the actuators separated by $\overrightarrow{x_{i j}}$. We also introduce the quantity $N\left(\overrightarrow{x_{i j}}\right)$, equal to the number of couples of actuators separated by $\overrightarrow{x_{i j}}$. Instead of summing over indexes $(i, j)$, we will sum over vectors $\overrightarrow{x_{i j}}$ :

$$
C(\vec{\rho})=\frac{1}{G(\rho)} \sum_{\overrightarrow{x_{i j}}} c_{v}\left(\overrightarrow{x_{i j}}\right) N\left(\overrightarrow{x_{i j}}\right) A\left(\overrightarrow{x_{i j}}+\vec{\rho}\right)
$$

and we should notice that there is no more approximation in this expression than that of Eq. 21, since we chose $c_{v}\left(\overrightarrow{x_{i j}}\right) N\left(\overrightarrow{x_{i j}}\right)=\sum_{x_{i j}}<v_{i} v_{j}>$ as a definition.

The function $N\left(\overrightarrow{x_{i j}}\right)$ is the number of couples of actuators with a given separation $\overrightarrow{x_{i j}}$ : it is similar to the function $G(\vec{\rho})$. We point out that the 2 "separation vectors" $\overrightarrow{x_{i j}}$ and $\vec{\rho}$ are conformable/similar, as they both aim to span pupil shifts. The previous equation 22 can be interpreted as the convolution between $A(\vec{\rho})$, and a distribution composed of a 2-dimensional series of Dirac functions $\delta\left(\vec{u}-\overrightarrow{x_{i j}}\right) c_{v}(\vec{u}) N(\vec{u})$ defined for any pupil vector $\vec{u}=\overrightarrow{x_{i j}}$. As the spatial extension of the function $A(\vec{\rho})$ is small compared to the size of the pupil, we will introduce our fourth approximation and write

$$
C(\vec{\rho})=\frac{1}{S_{p}} \sum_{\overrightarrow{x_{i j}}} c_{v}\left(\overrightarrow{x_{i j}}\right) A\left(\overrightarrow{x_{i j}}+\vec{\rho}\right)
$$

where $S_{p}$ is the area covered by the periodicity pattern of actuators, because $S_{p}$ must be equal to $N\left(\overrightarrow{x_{i j}}\right) / G\left(\overrightarrow{x_{i j}}\right)$ : the ratio between the number of actuators separated by $\overrightarrow{x_{i j}}$ and the pupil area where those actuators can be found. Notice that Eq. 22 apparently contains no computational difficulty: the purpose or the interest of our last approximation could then be questioned. There is an important reason to that : in reality the division by $G(\vec{\rho})$ in Eq. 22 is problematic because $G(\rho) \rightarrow 0$ when $\rho \rightarrow D$ and the quantity diverges at $\rho=D$. This divergence is due to our second approximation, where we decided to ignore the limitation of the integration by the pupil function. Our last approximation actually aims to solve this issue.

To summarize, building a map $C(\vec{\rho})$ is achieved following this : a separation vector $\overrightarrow{x_{i j}}$ is chosen. All the covariance values corresponding to identical separations $\overrightarrow{x_{i j}}$ between all possible actuators are extracted from the covariance matrix $C_{v v}$, and averaged (note this average does not come from Eq. 22, but from the very first assumption about spatial stationarity of the phase). The function $A(\vec{\rho})$ is scaled by this average value, and added to the map $C(\vec{\rho})$ with a shift $\overrightarrow{x_{i j}}$. When all $\overrightarrow{x_{i j}}$ have been parsed, $D_{\text {tomo }}(\vec{\rho})$ is computed using Eq. 18 .

\section{COMPUTATION OF COVARIANCE MATRICES}

The wavefront sensors split the pupil into square sub-apertures, that can be back-projected onto the turbulent layers according to the geometry of the light beam (parallel beam for NGS, conic one for LGS) defined by the guide star. Following this, all the distances between any projected couple of sub-apertures can be computed, as well as their sizes.

\subsection{General computation}

The covariance matrix of measurements $C_{m m}$ is the sum of covariance matrices associated to each of the $L$ layers of altitude $h_{l}$, with $1 \leq l \leq L$.

$$
C_{m m}=\sum_{l=1}^{L} C_{m m}\left(h_{l}\right)
$$


The computation of a matrix $C_{m m}(h)$ of a single layer located at altitude $h$ is then the base of the computation. As each layer is independent, all the $C_{m m}(h)$ can be computed independently. Each element $(i, j)$ of the matrix refers to the covariance between the wavefront slope (either $x$ or $y$ direction) of one of the sub-apertures of one of the WFS, and the same for a different sub-aperture of another WFS. Both sub-apertures need to be projected onto the turbulent layer following back the geometry of the beam that illuminates each WFS. The wavefront slope measured by a given square sub-aperture will be approximated by the phase difference between the 2 mid-points of two opposite edges. Using the binomial expansion

$$
2(A-a)(B-b)=-(A-B)^{2}+(A-b)^{2}+(a-B)^{2}-(a-b)^{2}
$$

we can rewrite the covariance of the phase difference using the phase structure function

$$
2<[\phi(A)-\phi(a)][\phi(B)-\phi(b)]>=-D_{\phi}(A B)+D_{\phi}(A b)+D_{\phi}(a B)-D_{\phi}(a b)
$$

with $A$ and $a 2$ midpoints of 2 opposite segments of a subaperture (same for $B$ and $b$ ), and where notations $A B$, $A b$, etc, stand for the distances between points $A$ and $B, A$ and $b$, etc. $D_{\phi}$ is the phase structure function (see paragraph 4.2). So, each element of the matrix has its own expression, which consists in computing distances between mid-points of segments of sub-apertures projected onto layers, and applying Eq. 26. This way of computing covariance matrices is ideally suited for parallel computation.

\subsection{The phase structure function with finite $L_{0}$}

A last dodgy detail is the computation of the phase structure function with a finite outer scale. For fully developed turbulence (infinite outer scale $L_{0}$ ), the phase structure function is given by

$$
D_{\phi}(r)=2\left(\frac{24}{5} \Gamma(6 / 5)\right)^{5 / 6}\left(r / r_{0}\right)^{5 / 3}=6.88\left(r / r_{0}\right)^{5 / 3}
$$

A more complex expression in the case of a finite $L_{0}$ can be found in $\mathrm{R}$. Conan $\mathrm{PhD}$ thesis. ${ }^{18}$ He has derived the expression of the phase structure function when considering a Von-Karman spectrum. The expression is:

$$
D_{\phi}(r)=k_{1} r_{0}^{-5 / 3} L_{0}^{5 / 3}\left(k_{2}-(2 x)^{5 / 6} K_{5 / 6}(2 x)\right)
$$

with

$$
\left\{\begin{array}{l}
x=\pi r / L_{0} \\
k_{1}=\Gamma(11 / 6) 2^{1 / 6} \pi^{-8 / 3}\left(\frac{24}{5} \Gamma(6 / 5)\right)^{5 / 6} \\
k_{2}=\Gamma(5 / 6) / 2^{1 / 6}
\end{array}\right.
$$

and where $K_{5 / 6}(2 x)$ can be computed as follows:

$$
K_{5 / 6}(2 x)=1 / 2 \sum_{n=0}^{\infty} \frac{(-1)^{n}}{n !}\left(\Gamma(-n-5 / 6) x^{2 n+5 / 6}+\Gamma(-n+5 / 6) x^{2 n-5 / 6}\right)
$$

Taken as it is, this expression is not computer-friendly nor efficient, but one can rewrite it to get rid of any power function and any gamma function. Firstly, replacing the expression of $K_{5 / 6}(2 x)$ given in Eq. 30 into Eq. 28 involves a multiplication by $x^{5 / 6}$ that will enter under the sum and simplify, plus making one of the first term of the sum at $n=0$ to cancel out with $k_{2}$ : this simplification is important to avoid computation roundoff errors. Secondly, defining the following variables and series:

$$
\left\{\begin{aligned}
y & =x^{5 / 3} \\
X_{1} & =x^{2} \\
X_{n} & =X_{n-1} X_{1} \\
a_{0} & =\Gamma(-5 / 6) / 2^{1 / 6} \\
a_{n} & =\frac{a_{n-1}}{n(n+5 / 6)} \\
b_{0} & =\Gamma(5 / 6) / 2^{1 / 6} \\
b_{n} & =\frac{b_{n-1}}{n(n-5 / 6)}
\end{aligned}\right.
$$


allows us to write

$$
D_{\phi}(r)=-k_{1} r_{0}^{-5 / 3} L_{0}^{5 / 3}\left(a_{0} y+\sum_{n=1}^{\infty}\left(a_{n} y+b_{n}\right) X_{n}\right),
$$

that can easily be implemented in a computer, especially since series $a_{n}$ and $b_{n}$ do not depend on $r$ and can just be precomputed and hardcoded as static arrays. Limiting this sum to $n=10$ will provide a good accuracy until $r<0.75 L_{0}$. After this limit, the asymptotic expansion of $K_{5 / 6}(z)$ towards infinity can do the job:

$$
z^{5 / 6} K_{5 / 6}(z) \approx \sqrt{\frac{2}{\pi}} z^{1 / 3} \exp (z)\left(1+\frac{2}{9 z}-\frac{7}{81 z^{2}}+\frac{175}{2187 z^{3}}+\ldots\right)
$$

\section{EFFICIENT IMPLEMENTATION OF THE TOMOGRAPHIC RECONSTRUCTOR COMPUTATION}

\subsection{Reconstructor computation, case of LGS}

In the noiseless approach described in previous sub-section, the inversion of matrix $C_{m m}$ in Eq. 1 is not a true inversion, as the null space of $C_{m m}$ may not be empty. The latter is composed of modes (i.e., WFS measurements of a given phase) that cannot be introduced by atmospheric turbulence and have thus a null variance under the Kolmogorov statistics. Examples of such modes are given in ${ }^{19}$. Additionally, because the global tip and tilt of the wavefront cannot be measured with LGS ${ }^{20}$ the 2 tip-tilt modes from each LGS WFS measurements have to be filtered out which introduces more null eigenvalues. A naive approach is to invert $C_{m m}$ using diagonalization and filter out the weakest eigenvalues.

An alternative approach is to filter out the modes by introducing noise contribution in the covariance matrix. First, WFS data suffer from detection and readout noise that should be taken into account. This noise is uncorrelated between sub-apertures and is just added on the diagonal elements of the covariance matrix and, in the case of LGS measurements, on sub-diagonal elements because of the intrinsic correlation of $x$ and $y$ measurements in this case due to the elongation of the laser spot. This adds a constant term to all eigenvalues and thus partly regularizes the inversion. The number of these eigenvalues will increase with the number of degrees of freedom of the system as more complex modes becomes available to the WFS. However, whatever the number of modes, because we add a diagonal to the covariance matrix, they will be filtered out anyway. So this approach is valid for any covariance matrix size, i.e. any system dimensioning (in terms of number of WFS and number of samples in the pupil).

Remains the LGS tip-tilt modes that have to be removed because they are present in the turbulence but cannot be measured by the system. We propose to follow an approach to filter them out during the inversion process, already mentioned by (Tallon et al. 2010) ${ }^{21}$. We need to get rid of these modes, as if they were measured with a null signal to noise ratio, i.e. with very high noise. It is thus possible to model an uncorrelated noise component due to tip-tilt measurements by adding a constant value to all $x$ and all $y$ measurements covariances of all LGS WFS. This component will add to the eigenvalues of the original Kolmogorov tip-tilt modes. If the value is high enough, its inverse tends to zero which in turn filters out these modes during the inversion. The same process could be applied to other unseen modes such as defocus.

Figure 1 outlines the various terms added to $C_{m m}$. The latter is the covariance matrix of all measurements from all WFS. Each WFS probes different regions of the wavefront, integrated over the respective lines of sight, and with a regular sampling. Each WFS produces a set of $x$ and $y$ measurements for each probed region of the pupil. $C_{m m}$ is the covariance matrix of all $x$ and $y$ measurements of all WFS. It is symmetric and positive definite and composed of blocks corresponding to each couple of WFS and sub-blocks for $x$ and $y$. Thanks to this approach, the null space of the modified $C_{m m}$ covariance matrix is empty and we can perform direct inversion instead of having to diagonalize it.

This new efficient approach, based on Cholesky factorization, to compute both the tomographic reconstructor and as a by-product, the tomographic error for given observational conditions, provides the level of accuracy required. This is demonstrated in figure 2 where we compare the end product of our pseudo-analytical model: the Point Spread Function for one science channel obtained using the two approaches: Eig-TR and Chol-TR 


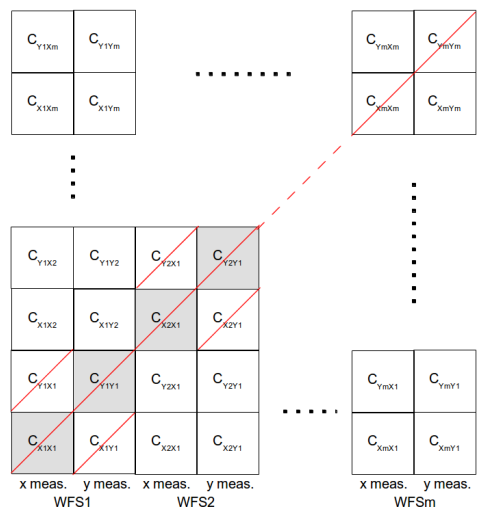

Figure 1. Structure of the $C_{m m}$ covariance matrix. In this case the first two WFS are using LGS. In red, the noise term added to all WFS. In grey, the constant component added to blocks of LGS WFS to filter out tip-tilt during the inversion.

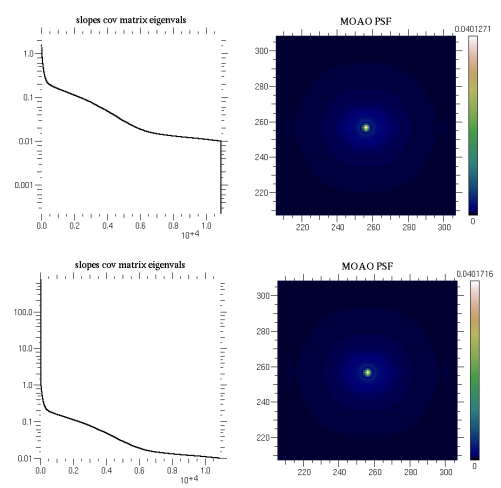

Figure 2. Left : eigen values of the $C_{m m}$ matrix to compute the tomographic reconstructor. Right the PSF obtained at the output of our pseudo-analytical model of a MOAO system. Top: naive approach using eigen values decomposition and mode filtering, bottom: regularized inversion.

after regularization of the $C_{m m}$ covariance matrix. This figure shows that the obtained image models using the two approaches are almost identical in terms of shape of the PSF and level of performance : Strehl ratios are respectively 0.04012 and 0.04017 . This accuracy is one to two orders of magnitude better than what is typically required for instrument design studies. On the left panel, we show the effect of the introducing of the two noise components on the eigenvalues of the $C_{m m}$ covariance matrix. In the top left panel, we see the drop at the end of the eigenvalues distribution corresponding to non-atmospheric modes and the filtered LGS tip-tilt modes. In the bottom left panel, the eigen values are shown after noise introduction: the weakest eigenvalues tend asymptotically to a given noise level and tip-tilt modes are no longer filtered but artificially amplified (largest eigen values) allowing direct inversion through Cholesky factorization.

\subsection{Efficient implementation}

In order to assess the maximum performance achievable for the computation of the tomographic reconstructor, we used the Matrices Over Runtime System at Exascale* (MORSE) ${ }^{22}$ project, a high-performance numerical library for solving large dense (and sparse) linear algebra problems on multicore systems with hardware accelerators. The goal of the MORSE project is to design linear algebra methods that achieve the fastest possible time to an accurate solution on large-scale multicore systems with hardware accelerators, using all the processing power that future high end homogeneous and heterogenous systems can make available. This framework describes matrix algorithms at a high level of abstraction and delegates the task execution order to a dynamic runtime system. As we use CUDA GPUs as accelerators, the StarPU ${ }^{23}$ Dynamic Runtime System was used. StarPU ${ }^{23}$ proposes a more productive and portable approach to dynamically schedule computational tasks simultaneously on CPU cores and on CUDA devices.

The tomographic reconstructor is calculated through four computational stages, that are extensively described in (Charara et al. 2014) ${ }^{24}$. The covariance matrix is generated from the system and turbulence parameters. It is first processed using the Cholesky factorization. The resulting triangular factor is then inverted. The inverted factor is then multiplied in-place by its transpose. The last stage consists of multiplying the final triangular matrix by a rectangular input matrix generated from the system and turbulence parameters. The core kernels of all stages are based on Level 3 BLAS operations and are therefore compute-bound. In this section, we show the results on the largest shared-memory machine with multi-GPUs we could access at the time of this writing. The system is representative of a vast class of servers and workstations commonly used for computationally intensive workloads.

\footnotetext{
*http://icl.cs.utk.edu/morse/
} 


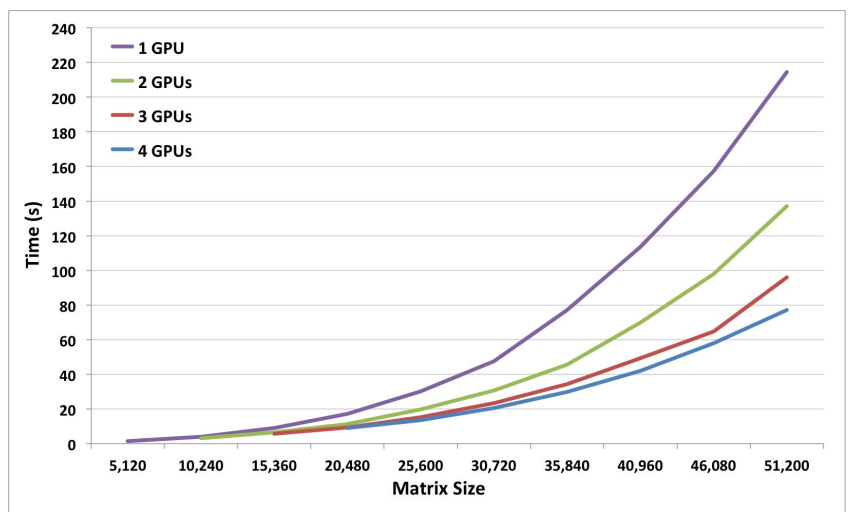

Figure 3. Performance of the tomographic reconstructor computation using regularized inversion in time (seconds).

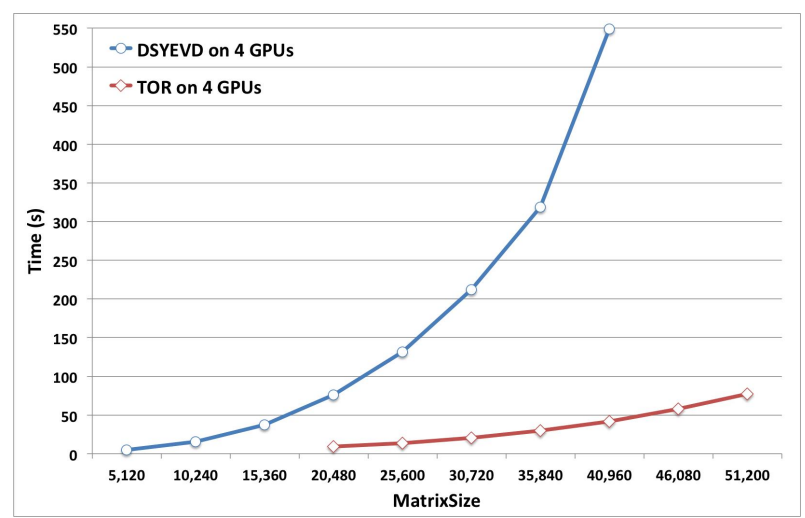

Figure 4. Performance comparison of the tomographic reconstructor computation using regularized inversion and diagonalization in time (seconds).

All calculations are performed in double precision floating-point arithmetic. The experiment ${ }^{24}$ were run on a dual-socket Intel(R) Xeon(R) CPU E5-2650 of eight cores each, running at $2.00 \mathrm{GHz}$, with a total of $64 \mathrm{~GB}$ of main memory. The theoretical peak is $256 \mathrm{Gflop} / \mathrm{s}$ (or $16 \mathrm{Gflop} / \mathrm{s}$ per core). The system is equipped with four NVIDIA Kepler K20c GPUs with ECC on. Each GPU has a peak performance of $1.3 \mathrm{Tflop} / \mathrm{s}$ with a sustained DGEMM peak at 1.1 Tflop/s. We compile the development branch of MORSE, StarPU v1.1.0 and MAGMA v1.4.1 with Intel compilers v13.0.1 and link against the Intel MKL (composer 2013).

Figure 3 shows timings in seconds of the tomographic reconstructor computation on homogeneous multicore as well as heterogenous environment with multi-GPUs. The time increases as the problem size raises. The homogenous implementation on 16 cores is overwhelmed by the compute intensity of the overall simulation and can not keep up with the high level of concurrency. Adding a single GPU drastically reduces the elapsed time. The time to solution decreases even further when adding more GPUs. It takes around 77 seconds to the tomographic reconstructor for a problem size of 51200 .

Figure 4 shows the timing comparison of the tomographic reconstructor based on the diagonalization method and the Cholesky factorization on four GPUs. Regularizing the covariance matrix pays off with a $13 \times$ speed-up compared to the diagonalization method.

\section{RESULT EXAMPLE}

The following example shows a PSF computed on a $42 \mathrm{~m}$ telescope for a configuration with 6 LGS positioned on a circle of 1.7 arcmin radius, and a NGS 40 arcsec off-axis, with $r_{0}=12.9 \mathrm{~cm}$. We are using a 9-layer turbulence atmospheric profile. We are using a $65 \times 65$ actuator DM, and all the WFS have $64 \times 64$ sub-apertures. The sodium layer altitude is $100 \mathrm{~km}$, with a gaussian profile of $5 \mathrm{~km} \mathrm{FWHM.} \mathrm{The} \mathrm{noise} \mathrm{level} \mathrm{corresponds} \mathrm{to} \mathrm{faint}$ stars, with $300 \mathrm{~nm}$ rms noise per sub-aperture. It takes into account the elongation of the LGS spots and the subsequent correlation of the noise due to this elongation, and its natural optimization in the reconstructor.

The following profile has been obtained on a 32 cores CPU system at $2.2 \mathrm{GHz}$, the full GPU version of the code being currently integrated.

\begin{tabular}{ll}
\hline Operation & Time \\
\hline \hline Covariance matrix generation & $73 \mathrm{~s}$ \\
Interaction matrix and inversion & $9.9 \mathrm{~s}$ \\
computation of $R$ and $C_{e e}$ & $663 \mathrm{~s}$ \\
Propagation from $C_{e e}$ to $<v_{i} v_{j}>1.9 \mathrm{~s}$ \\
$D_{\text {fit }}(\rho), D_{\mathrm{bw}}(\rho)$ & $0.2 \mathrm{~s}$ \\
PSF reconstruction & $14 \mathrm{~s}$ \\
\hline TOTAL & 12.7 minutes \\
\hline
\end{tabular}




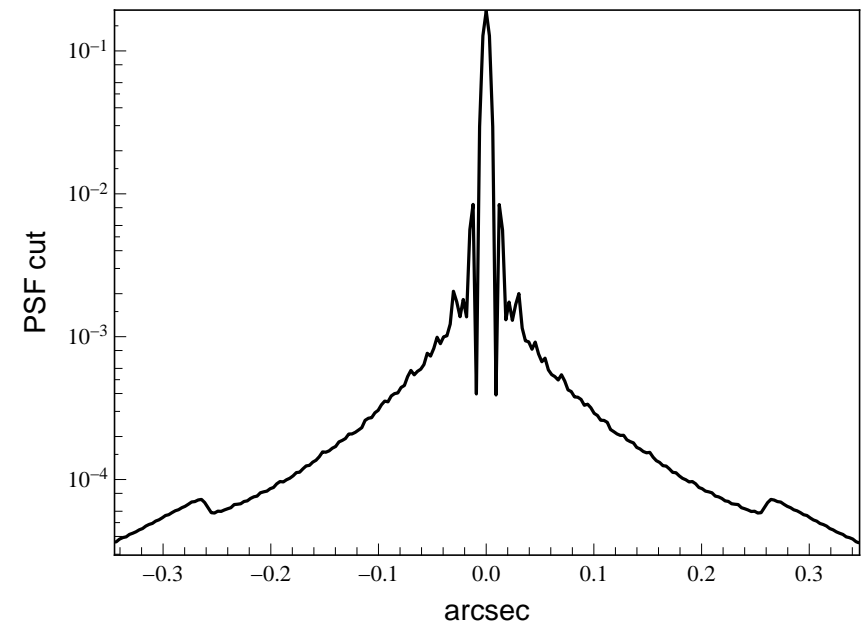

Figure 5. Cut of the simulated PSF normalized as a SR, versus arcseconds.

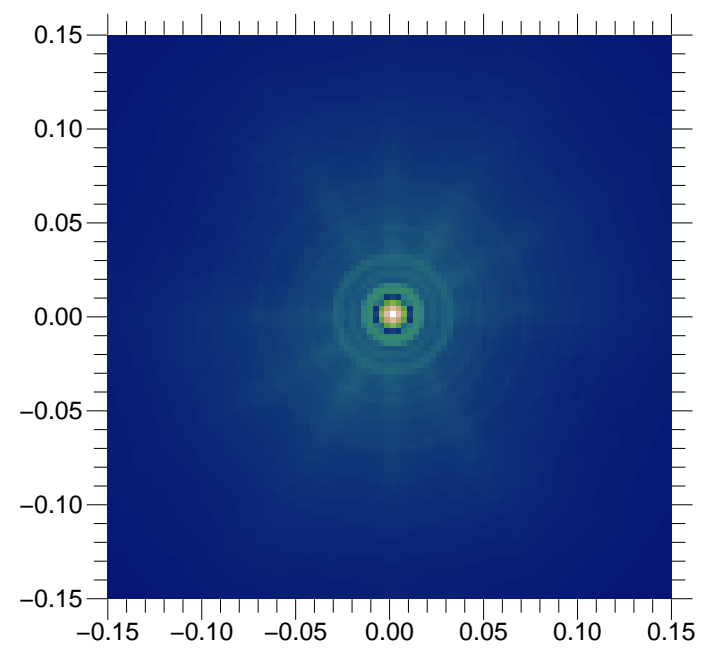

Figure 6. Image of the simulated PSF. Telescope $42 \mathrm{~m}$, 6 LGS on a 1.7 arcmin radius circle, 1 NGS $40 "$ off-axis.

Now, some additional simulations can be achieved varying the wavelength, the seeing, etc. This table shows the extra amount of time required for obtaining a supplementary PSF with those new conditions :

\begin{tabular}{ll}
\hline New simulation & Time \\
\hline \hline PSF at another wavelength & $+0.1 \mathrm{~s}$ each \\
PSF at different seeings & $+15 \mathrm{~s}$ each \\
PSFs at different wind speed, or gain, or sampling frequency & $+5 \mathrm{~s}$ each \\
PSF with another $C_{n}^{2}(h)$ profile (keeping same $\left.R\right)$ & $+2 \mathrm{mn}$ each \\
PSF somewhere else in the field & $+1 \mathrm{mn}$ each \\
\hline
\end{tabular}

\section{CONCLUSION}

We have developped a simulation tool for estimating the PSF obtained by a tomographic AO system, either LTAO or MOAO, i.e. optimized for a particular direction of interest. Out tools simulate the tomographic error, the noise effects, the aliasing, the bandwidth and fitting errors. It takes accurately into account the sampling of WFS, the edge effects of the pupil, etc, because it is based on a precise matrix description of the tomographic scheme. Not only the tomographic analysis allows us to simulate the performance of the system in the context of design trade-off studies, but also it could allow us to operate the future system as the tomographic reconstructor is the central component of the real-time controller.

The simulation is a merging of different techniques : 1) a technique for computing covariance matrices of measurements, 2) a matrix analysis for the tomographic, noise and aliasing effects, 3) a more classical pseudoanalytical analysis for the bandwidth and fitting errors and 4) a PSF reconstruction technique to transform the covariance matrices of the tomographic error into a phase structure function. We have developed this new way of reconstructing the PSF in order to cope with the very large problem size due to ELTs. The speedup factor of the PSF reconstruction step is so large that it can be achieved within a few seconds on any classical laptop today. Once again, we emphasize that this technique could again be used on-sky on a real system. Finally, once we have ended up with phase structure functions of all sources of errors, a couple of Fourier transforms allows us to retrieve the PSF.

Last but not least, most of the algorithms we propose here are specifically oriented for a parallel-computing implementation. In particular the covariance matrices of WFS measurements can benefit of incredible speedup factors depending on the parallel architecture of the machine it is ran on. But the most impressive advance in parallel computing and code pipelining has been done around the inversion of the covariance matrices for computing the tomographic reconstructor, and all the matrix operations required around it. The total computation time for a full simulation with 50000 measurements using 4 GPUs is less than 2 minutes. 
Knowing that the very same kind of operation will need to be done on real systems one day, we think our approach -both algorithms and implementation scheme- opens the right way to it.

\section{ACKNOWLEDGMENTS}

This work is partially supported by the ANR grant ANR-12-MONU-0022 of the French Ministry of Research. and partially by the European Commission Framework Programme 7, OPTICON, Grant agreement no: 312430 (WP1 Towards Adaptive Optics for the European Extremely Large Telescope), as well as CNRS/INSU, Observatoire de Paris, and Université Paris Diderot - Paris 7.

The authors would like to thank NVIDIA for their continuous support and hardware donations. The authors would like also to thank INRIA Bordeaux Runtime Team for the insightful discussions on optimizing the StarPU dynamic scheduler.

\section{REFERENCES}

[1] Cuby, J., Morris, S., Fusco, T., et al., "EAGLE: a MOAO fed multi-IFU NIR workhorse for E-ELT," in [Ground-based and Airborne Instrumentation for Astronomy III], Proc. SPIE 7735 (2010).

[2] Rousset, G., Fusco, T., Assémat, F., et al., "EAGLE MOAO system conceptual design and related technologies," in [Adaptive Optics Systems II], B. Ellerbroek, M. Hart, N. Hubin, P. Wizinowich, ed., Proc. SPIE 7736 (2010).

[3] Hammer, F., Sayède, F., Gendron, E., et al., "The FALCON Concept: Multi-Object Spectroscopy Combined with MCAO in Near-IR," in [Scientific Drivers for ESO Future VLT/VLTI Instrumentation], J. Bergeron \& G. Monnet, ed., Proc. of the ESO Workshop, 139 (2002).

[4] Evans, C. J., Barbuy, B., Bonifacio, P., et al., "Multi-object spectroscopy with the European ELT: scientific synergies between EAGLE and EVE," in [Ground-based and Airborne Instrumentation for Astronomy IV], Proc. SPIE 8446 (2012).

[5] Puech, M., Disseau, K., \& Pentericci, L., "Simulations of high-z galaxy observations with an E-ELT/MOS," in [ $3^{\text {rd }}$ AO4ELT conference], Esposito, S. \& Fini, L., eds., Adaptive Optics for Extremely Large Telescopes, INAF - Osservatorio Astrofisico di Arcetri, Florence, Italy (2013).

[6] Rigaut, F. J., Véran, J.-P., \& Lai, O., "Analytical model for Shack-Hartmann-based adaptive optics systems," in [Adaptive Optical System Technologies], D. Bonaccini \& R. K. Tyson, ed., Proc. SPIE 3353, 1038-1048 (1998).

[7] Jolissaint, L., Véran, J.-P., \& Conan, R., "Analytical modeling of adaptive optics: foundations of the phase spatial power spectrum approach," JOSA A 23, 382-394 (2006).

[8] Jolissaint, L., "Synthetic modeling of astronomical closed loop adaptive optics," Journal of the European Optical Society - Rapid publications, 5, 100555 (2010).

[9] Wallner, E. P., "Optimal wave-front correction using slope measurements," JOSA (1917-1983) 73, 1771 (1983).

[10] Fusco, T., Conan, J., Rousset, G., Mugnier, L. M., \& Michau, V., "Optimal wave-front reconstruction strategies for multiconjugate adaptive optics," JOSA A 18, 2527-2538 (2001).

[11] Vidal, F., Gendron, E., \& Rousset, G., "Tomography approach for multi-object adaptive optics," JOSA A 27, A253-A264 (2010).

[12] Gendron, E., Vidal, F., Brangier, M., et al., "MOAO first on-sky demonstration with CANARY," Astron. 83 Astrophys. 529, L2 (2011).

[13] Morris, T., Gendron, E., Basden, A., et al., "Multiple Object Adaptive Optics: Mixed NGS/LGS tomography," in [ $3^{r d}$ AO4ELT conference], Esposito, S. \& Fini, L., eds., Adaptive Optics for Extremely Large Telescopes, INAF - Osservatorio Astrofisico di Arcetri, Florence, Italy (2013).

[14] Vidal, F., Gendron, E., Rousset, G., et al., "Analysis of on-sky MOAO performance of CANARY using natural guide stars," Astron. \&5 Astrophys. in press (2014).

[15] Roddier, F., "The effects of atmospheric turbulence in optical astronomy," in [Progress in optics], Wolf, E., ed., 19, 281-376, North-Holland Publishing Co., Amsterdam (1981). 
[16] Véran, J.-P., Rigaut, F., Maître, H., \& Rouan, D., "Estimation of the adaptive optics long-exposure pointspread function using control loop data.," JOSA A 14, 3057-3069 (1997).

[17] Gendron, E., Clénet, Y., Fusco, T., \& Rousset, G., "New algorithms for adaptive optics point-spread function reconstruction," Astron. ES Astrophys. 457, 359-363 (2006).

[18] Conan, R., Modélisation des effets de l'échelle externe de cohérence spatiale du front d'onde pour l'observations à Haute Résolution Angulaire en Astronomie, $\mathrm{PhD}$ thesis, Université de Nice, Nice (2000).

[19] Gendron, E. \& Léna, P., "Astronomical adaptive optics. 1: Modal control optimization," Astron. \&3 Astrophys. 291, 337-347 (1994).

[20] Rigaut, F. \& Gendron, E., "Laser guide star in adaptive optics - The tilt determination problem," Astron. 83 Astrophys. 261, 677-684 (1992).

[21] Tallon, M., Tallon-Bosc, I., Béchet, C., et al., "Fractal iterative method for fast atmospheric tomography on extremely large telescopes," in [Adaptive Optics Systems II], B. Ellerbroek, M. Hart, N. Hubin, P. Wizinowich, ed., Proc. SPIE $\mathbf{7 7 3 6}$ (2010).

[22] MORSE, "MORSE, Matrix Over Runtime Systems at Exascale, http://icl.cs.utk.edu/morse/," (2014).

[23] Augonnet, C., Thibault, S., Namyst, R., \& Wacrenier, P., "StarPU: A unified platform for task scheduling on heterogeneous multicore architectures," Concurrency Computat. Pract. Exper. 23, 187-198 (2011).

[24] Charara, A., Ltaief, H., Gratadour, D., et al., "Pipelining computational stages of the tomographic reconstructor for multi-object adaptive optics on a multi-gpu system," in [ACM International Conference for High Performance Computing, Networking, Storage and Analysis 2014], (2014). 